\title{
Observation of the first hidden-charm strange tetraquark at BESIII
}

\author{
Jingyi Zhao* (on behalf of the BESIII Collaboration) \\ Institute of High Energy Physics, Chinese Academy of Sciences, 100049, China \\ E-mail: zhaojy@ihep.ac.cn
}

In the last ten years, a whole set of new particles that do not fit into the constituent quark model has populated the mass region above the open-charm threshold. The Beijing Spectrometer (BESIII), installed at the Beijing Electron Positron Collider (BEPCII), can access these states both in electron-positron annihilations and from the decays of charmonium(-like) states. In this proceeding, the discovery of the $Z_{c s}(3985)^{-}$at BESIII with a mass of $3982.5_{-2.6}^{+1.8} \pm 2.1 \mathrm{MeV} / c^{2}$ will be addressed. The resonance was observed by analyzing data collected at five center-of-mass energy points in the range from $4.628 \mathrm{GeV}$ to $4.698 \mathrm{GeV}$, with a total integrated luminosity of $3.7 \mathrm{fb}^{-1}$. The observed properties of the $Z_{c s}(3985)^{-}$makes it a strong candidate for the predicted open-strange charmonium-like tetraquark with the minimal structure $c \bar{c} s \bar{u}$.

The 22nd Particles and Nuclei International Conference

5-10 September, 2021

Lisbon, Portugal

${ }^{*}$ Speaker. 


\section{Introduction}

The Quantum Chromodynamics (QCD) theory [1, 2] predicted the existence of exotic hadrons in addition to the conventional meson and baryon. Exotic hadrons include e.g. glueball, hybrid, molecule, multi-quark state. Searching for exotic states therefore provides a test of QCD. It provides new insights into internal structure and dynamics of hadrons. In recent years, some unconventional $X Y Z$ particles [3] have been discovered experimentally. At BESIII, we have observed a series of $Z_{c}$ states [4-11], which are non-strange hidden-charm tetraquark candidates. Assuming $\mathrm{SU}(3)$ flavor symmetry, if we replace the $u$ or $d$ quark in $Z_{c}$ with an $s$ quark, we could expect the existence of a $Z_{c s}$ [12]. Theoreticaly prediction shows that the $Z_{c s}$ could decay into e.g. $K J / \psi$, $D_{s} D^{*}, D_{s}^{*} D$. Taking the $Z_{c}$ state as a reference, the decay rate of the $Z_{c s}$ to open charm final state is expected to be larger than the decay rate to hidden charm final state. Hence, a promising method to search for the $Z_{c s}$ state is through its decays to $D_{s} D^{*}$ and $D_{s}^{*} D$. At BESIII, we searched for $Z_{c s}^{-}$ through $e^{+} e^{-} \rightarrow K^{+} Z_{c s}^{-}, Z_{c s}^{-} \rightarrow\left(D_{s}^{-} D^{* 0}+D_{s}^{*-} D^{0}\right)$.

\section{Observation of the $Z_{c S}(3985)^{-}$at BESIII}

BEPCII extended the energy limit to $4.7 \mathrm{GeV}$ in 2019-2020. With the BESIII detector, we have collected $3.7 \mathrm{fb}^{-1}$ data accumulated from $4.628 \mathrm{GeV}$ to $4.698 \mathrm{GeV}$, which were used to search for $Z_{c s}^{-}$[13]. A partial reconstruction method is implemented to study $e^{+} e^{-} \rightarrow K^{+}\left(D_{s}^{-} D^{* 0}+\right.$ $\left.D_{s}^{*-} D^{0}\right)$. Only the bachelor $K^{+}$and the $D_{s}^{-}$are reconstructed. The signature in the recoil mass spectrum of $K^{+} D_{s}^{-}$is used to identify the signal process. The $Z_{c s}^{-}$is searched for in the recoil side of $K^{+}$. The $D_{s}^{-}$is reconstructed with two tag modes: $D_{s}^{-} \rightarrow K_{S}^{0} K^{-}$and $D_{s}^{-} \rightarrow K^{+} K^{-} \pi^{-}$, which have large branching fractions. For the decay $D_{s}^{-} \rightarrow K^{+} K^{-} \pi^{-}$, to further improve the signal purity, we only retain the $D_{s}^{-}$candidates consistent with $D_{s}^{-} \rightarrow \pi^{-} \phi, \phi \rightarrow K^{+} K^{-}$, and $D_{s}^{-} \rightarrow$ $K^{-} K^{*}(892), K^{*}(892) \rightarrow K^{+} \pi^{-}$.

Figure 1 shows the invariant mass distributions of $M\left(K^{+} K^{-} \pi^{-}\right)$and $M\left(K_{S}^{0} K^{-}\right)$at $\sqrt{s}=$ $4.681 \mathrm{GeV}$, in which $D_{s}^{-}$peaks are clearly evident. Figure 2 shows the recoil mass distribution $R M\left(K^{+} D_{s}^{-}\right)+M\left(D_{s}^{-}\right)-m\left(D_{s}^{-}\right)$. The original distribution $R M\left(K^{+} D_{s}^{-}\right)$is corrected by the nominal mass in PDG $\left(m\left(D_{s}^{-}\right)\right)$. The new variable $R M\left(K^{+} D_{s}^{-}\right)+M\left(D_{s}^{-}\right)-m\left(D_{s}^{-}\right)$provides improved resolution. A clear peak is seen in this distribution at the nominal $D^{* 0}$ mass (1-C kinematic fit is not performed). This peak corresponds to the final state $K^{+} D_{s}^{-} D^{* 0}$. There is also a contribution from $K^{+} D_{s}^{*-} D^{0}$, which appears to be a broader structure beneath the $K^{+} D_{s}^{-} D^{* 0}$ signal.

To estimate the shape of the combinatorial background, we use wrong-sign combinations of $D_{s}^{-}$and $K^{-}$candidates, rather than the right-sign $D_{s}^{-}$and $K^{+}$candidates. The wrong sign distribution agrees with the data distribution in the sideband regions. No peaking background is observed in the wrong-sign events. The number of background events within the signal region is estimated to be $N_{b}=282.6 \pm 12.0$ from the fit to the sideband data with a linear function. The slope of the function is determined from the wrong-sign data. In addition, the wrong-sign events would be used to represent the combinatorial background distribution in the recoil mass of $K^{+}$. This technique has been well validated by MC simulations and data sideband events.

Figure 3 shows the recoil mass distribution $R M\left(K^{+}\right)$. Figure 3 shows the same distribution at $4.681 \mathrm{GeV}$ after subtracting the combinatorial background. An enhancement is evident in the 

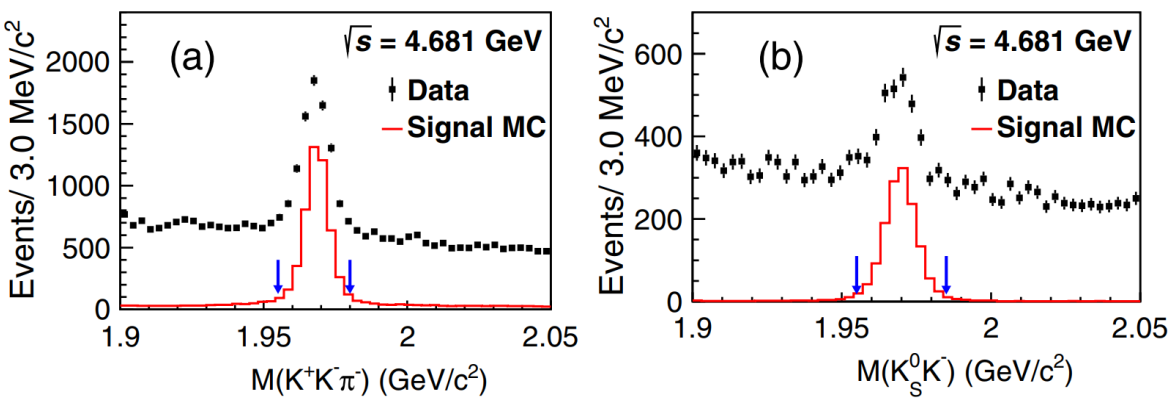

Figure 1: Distributions of the invariant mass $M\left(K^{+} K^{-} \pi^{-}\right)$and $M\left(K_{S}^{0} K^{-}\right)$in data and MC simulations at $\sqrt{s}=4.681 \mathrm{GeV}$. The $Z_{c s}(3985)^{-}$signal MC component is normalized to the observed $D_{s}^{-}$yield in dat. Arrows indicate the mass region requirements.

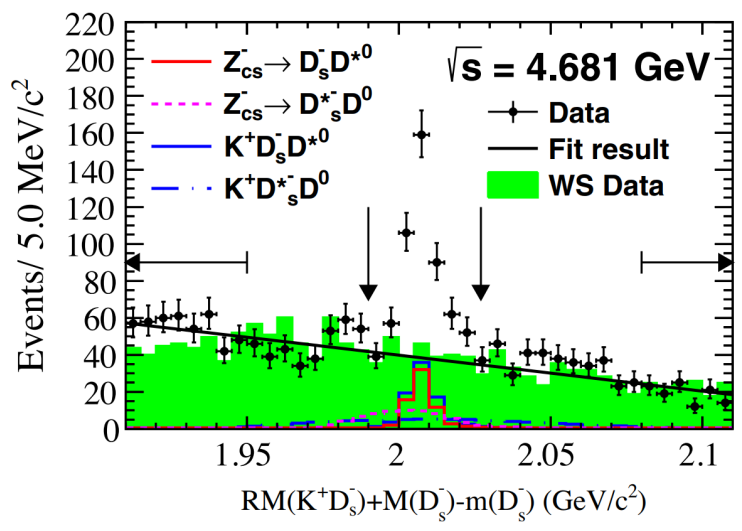

Figure 2: Distribution of the $K^{+} D_{s}^{-}$recoil mass in data and signal MC samples at $\sqrt{s}=4.681 \mathrm{GeV}$. Horizontal arrows indicate the sidebands and vertical arrows indicate the signal region. The magnitudes of the three-body nonresonant processes and $Z_{c} s(3985)^{-}$signal processes are scaled arbitrarily. The histogram of wrong-sign events is scaled by a factor of 1.18 to match the sideband data.

region below $4 \mathrm{GeV}$. Some processes containing $D_{s}^{* *}$ states have potential contributions to the $R M\left(K^{+}\right)$spectrum. For example, $e^{+} e^{-} \rightarrow D_{s 1}^{*}(2536)^{+}\left(K^{+} D^{* 0}\right) D_{s}^{-}, e^{+} e^{-} \rightarrow D_{s 2}^{*}(2573)^{+}\left(K^{+} D^{0}\right) D_{s}^{*-}$, and $e^{+} e^{-} \rightarrow D_{s 1}^{*}(2700)^{+}\left(K^{+} D^{* 0}\right) D_{s}^{-}$have exactly the same final state as our signal channel, which could be peaking background in the $R M\left(K^{+}\right)$spectrum. We check the $D_{s}^{* *}$ states listed in PDG one by one, and estimate their production cross sections by studying several control samples. The contribution from processes containing $D_{s}^{* *}$ states would be considered in the fit of $R M\left(K^{+}\right)$distribution. We have checked the interference between any two processes containing $D_{s}^{* *}$ states, and the interference between the process containing a $D_{s}^{* *}$ state and non-resonance process. The study shows that the interference effect could not produce a narrow peak in the $R M\left(K^{+}\right)$spectrum.

The fact that no known process can explain the observed enhancement in the $R M\left(K^{+}\right)$spectrum, indicates that the structure corresponds to a new state, denoted $Z_{c s}(3985)^{-}$. A simultaneous unbinned maximum likelihood fit is applied to $R M\left(K^{+}\right)$spectrum from data taken at five center-of-mass energies. The $Z_{c s}(3985)^{-}$signal is described with a S-wave Breit-Wigner with mass dependet width with phase-space factor. The potential interference effect between any two components is neglected. From this fit, we get the resonance parameters of this new structure to 


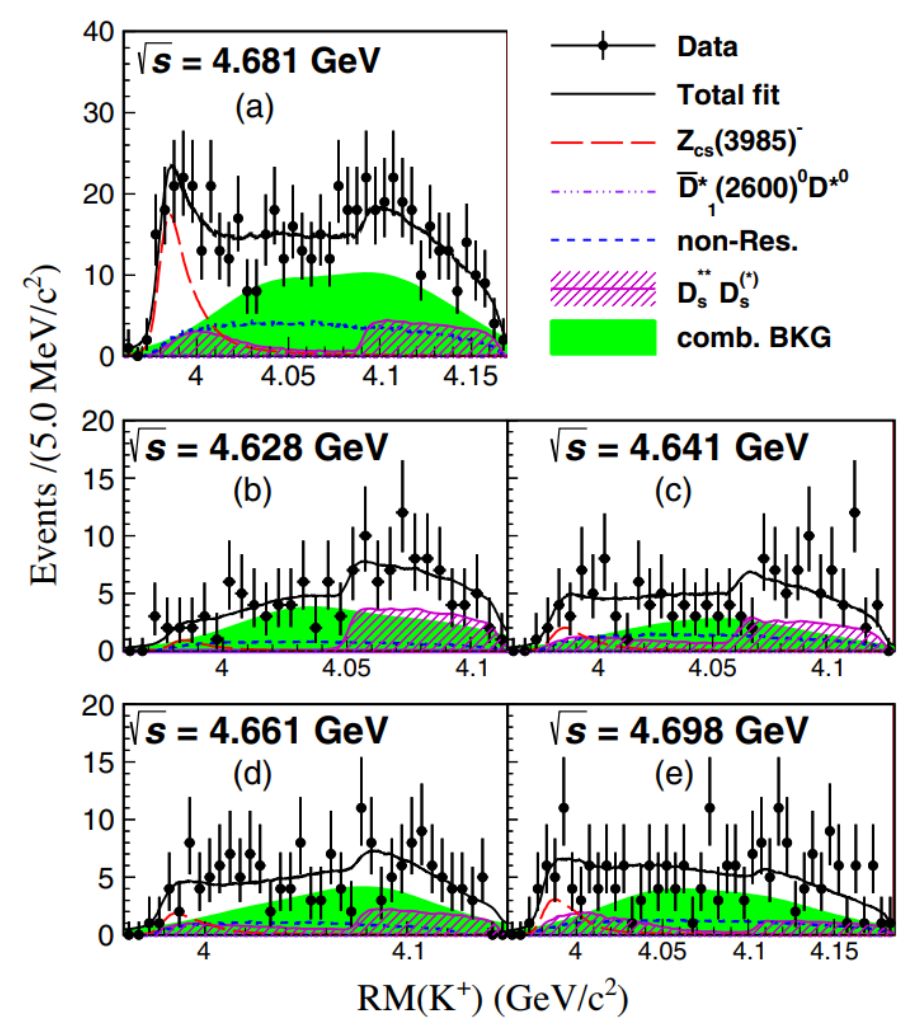

Figure 3: Simultaneous unbinned maximum likelihood fit to the $K^{+}$recoil-mass spectra in data at $\sqrt{s}=$ $4.628,4.641,4.661,4.681$, and $4.698 \mathrm{GeV}$. Note that the size of the $D^{* 0} \bar{D}_{1}^{*}(2600)^{0}\left(\rightarrow D_{s}^{-} K^{+}\right)$component is consistent with zero.

be $m_{0}\left(Z_{c s}(3985)^{-}\right)=\left(3985.2_{-2.0}^{+2.1} \pm 1.7\right) \mathrm{MeV} / c^{2}$ and $\Gamma_{0}\left(Z_{c s}(3985)^{-}\right)=\left(13.8_{-5.2}^{+8.1} \pm 4.9\right) \mathrm{MeV}$, which correspond to the pole position parameters $m_{\text {pole }}\left(Z_{c s}(3985)^{-}\right)=\left(3982.5_{-2.6}^{+1.8} \pm 2.1\right) \mathrm{MeV} / c^{2}$ and $\Gamma_{\text {pole }}\left(Z_{c S}(3985)^{-}\right)=\left(12.8_{-4.4}^{+5.3} \pm 3.0\right) \mathrm{MeV}$. The significance of $Z_{c s}(3985)^{-}$considering systematic uncertainties and look-elsewhere effect is evaluated to be $5.3 \sigma$. The mass of $Z_{c s}(3985)^{-}$ is only a few $\mathrm{MeV}$ higher than the threshold of $D_{s}^{-} D^{* 0}$ and $D_{s}^{*-} D^{0}$. It contains at least four quarks $c \bar{c} s \bar{u}$ and be a strong candidate for the predicted open-strange charmonium-like tetraquark state.

\section{Comparison with results from $\mathrm{LHCb}$}

The LHCb Collaboration observed a new structure from its decaying into the $J / \psi K^{+}$final state [14]. The structure is denoted as $Z_{c S}(4000)^{+}$, which has a pole mass of $4003 \pm 6_{-14}^{+4} \mathrm{MeV}$, and a pole width of $131 \pm 15 \pm 26 \mathrm{MeV}$. The masses of $Z_{c s}(3985)^{-}$and $Z_{c s}(4000)^{+}$are close, but the widths are quite different. The LHCb Collaboration tried to fix the mass and width of $Z_{c s}(4000)^{+}$ to values of $Z_{c s}(3985)^{-}$state, but the log-likelihood of the fit is much worse. Therefore, there is no evidence that the $Z_{c s}(4000)^{+}$is the same as the $Z_{c s}(3985)^{-}$.

\section{Summary}

The BESIII Collaboration studies the reactions $e^{+} e^{-} \rightarrow K^{+}\left(D_{s}^{-} D^{* 0}+D_{s}^{*-} D^{0}\right)$ based on $3.7 \mathrm{fb}^{-1}$ 
of data collected at $\sqrt{s}=4.628,4.641,4.661,4.681$, and $4.698 \mathrm{GeV}$. A new structure, which is denoted as $Z_{c s}(3985)^{-}$, is observed near the $D_{s}^{-} D^{* 0}$ and $D_{s}^{*-} D^{0}$ thresholds in the $K^{+}$recoil-mass spectrum. The significance of $Z_{c s}(3985)^{-}$is $5.3 \sigma$.

The quark composition of $Z_{c s}(3985)^{-}$is most likely $c \bar{c} s \bar{u}$. Hence, it would become the first $Z_{c s}$ tetraquark candidate observed. The cross section $\sigma^{B}\left[e^{+} e^{-} \rightarrow K^{+} Z_{c s}(3985)^{-}+\right.$c.c. $]$times branching fraction $\mathscr{B}\left(Z_{c s}(3985)^{-} \rightarrow\left(D_{s}^{-} D^{* 0}+D_{s}^{*-} D^{0}\right)\right.$ at five center-of-mass energies are also measured. Comparing with the $Z_{c s}(4000)^{+}$observed by the LHCb Collaboration, the $Z_{c s}(3985)^{-}$ is much narrower. There is no evidence that the $Z_{c s}(3985)^{-}$and $Z_{c s}(4000)^{+}$are the same structure.

\section{References}

[1] M. Gell-Mann, A schematic model of baryons and mesons, Phys. Lett. 8214 (1964).

[2] G. Zweig, An SU(3) model for strong interaction symmetry and its breaking, CERN-TH 41280 (1964).

[3] P.A. Zyla et al. (Particle Data Group), Review of particle physics, Prog. Theor. Exp. Phys. 2020, 083C01 (2020) and 2021 update.

[4] M. Ablikim et al. (BESIII Collaboration), Observation of a charged charmoniumlike structure in $e^{+} e^{-} \rightarrow \pi^{+} \pi^{-} J / \psi$ at $\sqrt{s}=4.26 \mathrm{GeV}$, Phy. Rev. Lett. 110252001 (2013).

[5] M. Ablikim et al. (BESIII Collaboration), Observation of $Z_{c}(3900)^{0}$ in $e^{+} e^{-} \rightarrow \pi^{0} \pi^{0} J / \psi$, Phy. Rev. Lett. 115112003 (2015).

[6] M. Ablikim et al. (BESIII Collaboration), Observation of a charged charmoniumlike structure $Z_{c}(4020)$ and search for the $Z_{c}$ (3900) in $e^{+} e^{-} \rightarrow \pi^{+} \pi^{-} h_{c}$, Phy. Rev. Lett. 111242001 (2013).

[7] M. Ablikim et al. (BESIII Collaboration), Observation of $e^{+} e^{-} \rightarrow \pi^{0} \pi^{0} h_{c}$ and a neutral charmoniumlike structure $Z_{c}(4020)^{0}$, Phy. Rev. Lett. 113212002 (2014).

[8] M. Ablikim et al. (BESIII Collaboration), Observatin of a charged $\left(D \bar{D}^{*}\right)^{ \pm}$mass peak in $e^{+} e^{-} \rightarrow \pi D \bar{D}^{*}$ at $\sqrt{s}=4.26 \mathrm{GeV}$, Phy. Rev. Lett. 112022001 (2014).

[9] M. Ablikim et al. (BESIII Collaboration), Observation of a neutral structure near the DD $\bar{D}^{*}$ mass threshold in $e^{+} e^{-} \rightarrow\left(D \bar{D}^{*}\right)^{0} \pi^{0}$ at $\sqrt{s}=4.226$ and $4.257 \mathrm{GeV}$, Phy. Rev. Lett. 115222002 (2015).

[10] M. Ablikim et al. (BESIII Collaboration), Observation of a charged charmoniumlike structure in $e^{+} e^{-} \rightarrow\left(D^{*} \bar{D}^{*}\right)^{ \pm} \pi^{\mp}$ at $\sqrt{s}=4.26 \mathrm{GeV}$, Phy. Rev. Lett. 112132001 (2014).

[11] M. Ablikim et al. (BESIII Collaboration), Observation of a neutral charmoniumlike state $Z_{c}(4025)^{0}$ in $e^{+} e^{-} \rightarrow\left(D^{*} \bar{D}^{*}\right)^{0} \pi^{0}$, Phy. Rev. Lett. 115182002 (2015).

[12] M.B. Voloshin, Strange hadrocharmonium, Phys. Lett. B 798, 135022 (2019).

[13] M. Ablikim et al. (BESIII Collaboration), Observation of a near-threshold structure in the $K^{+}$ recoil-mass spectra in $e^{+} e^{-} \rightarrow K^{+}\left(D_{s}^{-} D^{* 0}+D_{s}^{*-} D^{0}\right)$, Phy. Rev. Lett. 126102001 (2021).

[14] R. Aaij et al. (LHCb Collaboration), Observation of new resonance decaying to $J / \psi K^{+}$and $J / \psi \phi$, Phy. Rev. Lett. 127082001 (2021). 